\title{
Metric Operator in Pseudo-Hermitian Quantum Mechanics and the Imaginary Cubic Potential
}

\author{
Ali Mostafazadeh \\ Department of Mathematics, Koç University, \\ 34450 Sariyer, Istanbul, Turkey \\ amostafazadeh@ku.edu.tr
}

\begin{abstract}
We present a systematic perturbative construction of the most general metric operator (and positive-definite inner product) for quasi-Hermitian Hamiltonians of the standard form, $H=\frac{1}{2} p^{2}+v(x)$, in one dimension. We show that this problem is equivalent to solving an infinite system of iteratively decoupled hyperbolic partial differential equations in $(1+1)$-dimensions. For the case that $v(x)$ is purely imaginary, the latter have the form of a nonhomogeneous wave equation which admits an exact solution. We apply our general method to obtain the most general metric operator for the imaginary cubic potential, $v(x)=i \epsilon x^{3}$. This reveals an infinite class of previously unknown $\mathcal{C P} \mathcal{T}$ - as well as non- $\mathcal{C} \mathcal{P} \mathcal{T}$-inner products. We compute the physical observables of the corresponding unitary quantum system and determine the underlying classical system. Our results for the imaginary cubic potential show that, unlike the quantum system, the corresponding classical system is not sensitive to the choice of the metric operator. As another application of our method we give a complete characterization of the pseudo-Hermitian canonical quantization of a free particle moving in $\mathbb{R}$ that is consistent with the usual choice for the quantum Hamiltonian. Finally we discuss subtleties involved with higher dimensions and systems having a fixed length scale.
\end{abstract}

PACS number: 03.65.-w

Keywords: metric operator, pseudo-Hermitian, quasi-Hermitian, $\mathcal{P} \mathcal{T}$-symmetry, complex potential, quantization 


\section{Introduction}

In Quantum Mechanics the unitarity of the dynamics dictates that the Hamiltonian operator must be Hermitian (self-adjoint). Therefore a non-Hermitian operator $H$ cannot serve as the Hamiltonian for a unitary quantum system unless the Hilbert space is endowed with a different inner-product which renders $H$ Hermitian. Under rather general conditions, one can show that a necessary and sufficient condition for the existence of such an inner product is the reality of the spectrum of $H$, [1]. The most general inner product that makes $H$ Hermitian has the form

$$
\langle\cdot, \cdot\rangle_{\eta_{+}}:=\left\langle\cdot\left|\eta_{+}\right| \cdot\right\rangle
$$

where $\langle\cdot \mid \cdot\rangle$ is the defining inner product of the original (reference) Hilbert space $\mathcal{H}, \eta_{+}: \mathcal{H} \rightarrow \mathcal{H}$ is a positive-definite operator satisfying the pseudo-Hermiticity condition [2]

$$
H^{\dagger}=\eta_{+} H \eta_{+}^{-1}
$$

and $H^{\dagger}: \mathcal{H} \rightarrow \mathcal{H}$ is the adjoint of $H .^{1}$

The operator $\eta_{+}$, which is sometimes referred to as the metric operator, is the basic ingredient of a quantum theory based on the Hamiltonian $H$. It determines the inner product (11) of the physical Hilbert space $\mathcal{H}_{\text {phys }}$ and specifies the observables $O$ of the theory, [4, 5, 6, 17. Furthermore, it provides the necessary means to obtain an equivalent description of the system using a Hermitian Hamiltonian $h$ acting in $\mathcal{H}$. This in turn allows for the determination of the underlying classical system, [5, 6].

An important property of the metric operator, is that it is not unique [8, 9, 10, 11]. For example, the $\mathcal{C} \mathcal{P} \mathcal{T}$-inner product proposed in [12] can be conveniently expressed in the form (11) for a particular choice of $\eta_{+}$, 9, 13. As such, the $\mathcal{C P} \mathcal{T}$-inner product is not the only allowed choice for the inner product of the physical Hilbert space; it is merely an example of an infinite set of possible choices.

In [5, 6, 7] we have constructed specific metric operators for different $\mathcal{P} \mathcal{T}$-symmetric systems and examined the physical consequences and the classical limit of the quantum systems they define. In the present article we intend to address the issue of the non-uniqueness of the metric operator. In particular, we will offer a perturbative construction of the most general metric operator for the standard Hamiltonians in one dimension, and explore the consequences of adopting different metric operators for a given Hamiltonian operator.

Our method which is based on a variation of the approach of [14] has the following two advantages. $(i)$ It does not involve using a particular ansatz for each choice of the potential. Instead, it employs the well-established properties of $(1+1)$-dimensional hyperbolic partial differential equations. This makes it applicable for all potentials. ( $i i$ ) It is not restricted to producing the $\mathcal{C P} \mathcal{T}$-inner products; it yields the most general inner product that is capable of restoring the unitarity. In fact, as we will see for the particular example of the imaginary cubic potential, it reveals a new set of $\mathcal{C P} \mathcal{T}$-inner products that has escaped the analysis of [14].

\footnotetext{
${ }^{1}$ For any densely defined $H, H^{\dagger}$ is the unique operator satisfying $\left\langle\cdot \mid H^{\dagger} \cdot\right\rangle=\langle H \cdot \mid \cdot\rangle,[]$.
} 


\section{Perturbative Construction of the Metric Operator}

Consider a Hamiltonian of the form

$$
H=H_{0}+\epsilon H_{1},
$$

where $\epsilon$ is a real perturbation parameter and $H_{0}$ and $H_{1}$ are respectively Hermitian and antiHermitian $\epsilon$-independent operators. Suppose that for sufficiently small values of $\epsilon$ the Hamiltonian $H$ has a real spectrum and a complete set of eigenvectors, so that a positive-definite metric operator $\eta_{+}$exists [1]. Such an operator has a well-defined Hermitian logarithm. We shall let $Q:=-\ln \eta_{+}$, or alternatively

$$
\eta_{+}=e^{-Q},
$$

and employ (2) to obtain a perturbative expansion for $Q$.

The condition that for $\epsilon=0$, the inner product $\langle\cdot, \cdot\rangle_{\eta_{+}}$of $(\mathbb{1})$ reduces to $\langle\cdot \mid \cdot\rangle$, i.e., as $\epsilon \rightarrow 0$ we have $\eta_{+} \rightarrow 1$, justifies the following perturbation expansion for $Q$.

$$
Q=\sum_{j=1}^{\infty} Q_{j} \epsilon^{j},
$$

where $Q_{j}$ are $\epsilon$-independent Hermitian operators. A convenient tool for calculating the operators $Q_{j}$ is the Baker-Campbell-Hausdorff formula,

$$
e^{-Q} H e^{Q}=H+\sum_{k=1}^{\infty} \frac{1}{k !}[H, Q]_{k},
$$

where $[H, Q]_{1}:=[H, Q]$ and $[H, Q]_{k+1}:=\left[[H, Q]_{k}, Q\right]$ for all $k \in \mathbb{Z}^{+}:=\{1,2,3, \cdots\}$. As we show in Appendix A, we can use (6) to obtain the following infinite set of operator equations for $Q_{j}$.

$$
\left[H_{0}, Q_{j}\right]=R_{j},
$$

where $j \in \mathbb{Z}^{+}$is arbitrary and

$$
\begin{aligned}
& R_{j}:=\left\{\begin{array}{cc}
-2 H_{1} & \text { for } \quad j=1 \\
\sum_{k=2}^{j} q_{k} Z_{k j} & \text { for } j \geq 2,
\end{array}\right. \\
& q_{k}:=\sum_{m=1}^{k} \sum_{n=1}^{m} \frac{(-1)^{n} n^{k}}{k ! 2^{m-1}}\left(\begin{array}{c}
m \\
n
\end{array}\right), \\
&\left.Z_{k j}:=\sum_{\substack{s_{1}, s_{2}, \cdots, s_{k} \in \mathbb{Z}^{+} \\
s_{1}+\cdots+s_{k}=j}}\left[\left[\cdots\left[H_{0}, Q_{s_{1}}\right], Q_{s_{2}}\right], \cdots,\right], Q_{s_{k}}\right] .
\end{aligned}
$$

It is not difficult to see that the anti-Hermitian operators $R_{j}$ are uniquely determined by $H_{1}$, $Q_{1}, Q_{2}, \cdots, Q_{j-1}$. This shows that (7) may in principle be solved iteratively. For example 
setting $j=1,2,3,4$ and 5 , we respectively find

$$
\begin{aligned}
{\left[H_{0}, Q_{1}\right]=} & -2 H_{1}, \\
{\left[H_{0}, Q_{2}\right]=} & 0 \\
{\left[H_{0}, Q_{3}\right]=} & \frac{1}{12}\left[H_{0}, Q_{1}\right]_{3}=-\frac{1}{6}\left[H_{1}, Q_{1}\right]_{2}, \\
{\left[H_{0}, Q_{4}\right]=} & \frac{1}{12}\left(\left[\left[H_{0}, Q_{1}\right]_{2}, Q_{2}\right]+\left[\left[\left[H_{0}, Q_{1}\right], Q_{2}\right], Q_{1}\right]+\left[\left[H_{0}, Q_{2}\right], Q_{1}\right]_{2}\right) \\
= & -\frac{1}{6}\left(\left[\left[H_{1}, Q_{1}\right], Q_{2}\right]+\left[\left[H_{1}, Q_{2}\right], Q_{1}\right]\right), \\
{\left[H_{0}, Q_{5}\right]=} & -\frac{1}{120}\left[H_{0}, Q_{1}\right]_{5}+\frac{1}{12}\left(\left[\left[H_{0}, Q_{1}\right], Q_{2}\right]_{2}+\left[\left[H_{0}, Q_{2}\right], Q_{1}\right]_{2}+\left[\left[H_{0}, Q_{2}\right]_{2}, Q_{1}\right]+\right. \\
& {\left.\left[\left[H_{0}, Q_{1}\right]_{2}, Q_{3}\right]+\left[\left[\left[H_{0}, Q_{1}\right], Q_{3}\right], Q_{1}\right]+\left[\left[H_{0}, Q_{3}\right], Q_{1}\right]_{2}\right) } \\
= & \frac{1}{360}\left[H_{1}, Q_{1}\right]_{4}-\frac{1}{6}\left(\left[H_{1}, Q_{2}\right]_{2}+\left[\left[H_{1}, Q_{1}\right], Q_{3}\right]+\left[\left[H_{1}, Q_{3}\right], Q_{1}\right]\right),
\end{aligned}
$$

where we have made use of the fact that $q_{k}$ vanishes for even values of $k$.

Comparing (11) - (15) with operator equations obtained in 14 for $Q_{j}$, we see that the choice of the $\mathcal{C P} \mathcal{T}$-inner product corresponds to taking $Q_{2}=Q_{4}=\cdots=Q_{2 j}=\cdots=0$. Furthermore this choice restricts $Q_{2 j+1}$ to the solutions of (17) that are even in $x$ and odd in $p$. We will refrain from imposing such restrictions and try to obtain the most general solution of (17).

Setting $H_{0}=\frac{1}{2 m} p^{2}+v_{0}(x)$, where $v_{0}$ is a real-valued function, adopting units in which $m=\hbar=1$, and evaluating the matrix-elements of both sides of (17) in the $x$-representation, we find

$$
\left[-\partial_{x}^{2}+\partial_{y}^{2}+2 v_{0}(x)-2 v_{0}(y)\right]\left\langle x\left|Q_{j}\right| y\right\rangle=2\left\langle x\left|R_{j}\right| y\right\rangle .
$$

This is a nonhomogeneous hyperbolic partial differential equation in $(1+1)$-dimensions. In particular, for the cases that $v_{0}=0$, i.e., $H_{0}=\frac{1}{2} p^{2}$, it reduces to the $(1+1)$-dimensional nonhomogeneous wave equation,

$$
\left[-\partial_{x}^{2}+\partial_{y}^{2}\right]\left\langle x\left|Q_{j}\right| y\right\rangle=2\left\langle x\left|R_{j}\right| y\right\rangle
$$

whose general closed-form solution is well-known [15].

In summary, we have reduced the problem of determining $Q_{j}$ to the solution of a set of iteratively decoupled and well-known partial differential equations. In fact, because these equations only differ in the form of their nonhomogeneity, we can express their general solution as

$$
\left\langle x\left|Q_{j}\right| y\right\rangle=f_{j}(x, y)+\mathcal{Q}_{j}(x, y)
$$

where $f_{j}$ is the general solution of the homogeneous equation

$$
\left[-\partial_{x}^{2}+\partial_{y}^{2}+2 v_{0}(x)-2 v_{0}(y)\right] f_{j}(x, y)=0
$$

and $\mathcal{Q}_{j}$ is a particular solution of (16). The only restriction on $f_{j}$ and $\mathcal{Q}_{j}$ is that they should be selected in such a way that $\left\langle x\left|Q_{j}\right| y\right\rangle^{*}=\left\langle y\left|Q_{j}\right| x\right\rangle$, i.e., yield a Hermitian $Q_{j}$. We can adopt, without loss of generality, a particular solution satisfying the Hermiticity condition 
$\mathcal{Q}_{j}(x, y)=\mathcal{Q}_{j}(y, x)^{*}$. In this case $f_{j}(x, y)=f_{j}(y, x)^{*}$ and as a result the general form of $f_{j}$ is independent of $j{ }^{2}$

For the case that $H_{0}=\frac{1}{2} p^{2}, f_{j}$ is given by d'Alemert's formula [15]:

$$
f_{j}(x, y)=\varphi_{j}(x-y)+\chi_{j}(x+y),
$$

where $\varphi_{j}, \chi_{j}: \mathbb{R} \rightarrow \mathbb{C}$ are arbitrary twice differentiable functions. ${ }^{3}$ Because, according to (12), $R_{2}=0$, we can set $\mathcal{Q}_{2}(x, y)=0$ and use (18) and (201), to obtain

$$
\left\langle x\left|Q_{2}\right| y\right\rangle=\varphi_{2}(x-y)+\chi_{2}(x+y) .
$$

We can easily obtain the explicit form of the operator $Q_{2}$ using (21). The result is $Q_{2}=$ $\tilde{\varphi}_{2}(p)+\tilde{\chi}_{2}(p) \mathcal{P}$, where a tilde stands for the Fourier transform: $\tilde{F}(p):=\int_{-\infty}^{\infty} e^{-i p x} F(x) d x$, and $\mathcal{P}$ is the parity operator. The same reasoning shows that, whenever $H_{0}=\frac{1}{2} p^{2}$,

$$
Q_{j}=\mathcal{Q}_{j}+F_{j}+K_{j} \mathcal{P},
$$

where $j \in \mathbb{Z}^{+}$is arbitrary, $\mathcal{Q}_{j}$ is a particular solution of the operator equation (17), and $F_{j}=$ $\tilde{\varphi}_{j}(p)$ and $K_{j}=\tilde{\chi}_{j}(p)$ are any functions of $p$ that together with $\mathcal{Q}_{j}$ make $Q_{j}$ Hermitian. ${ }^{4}$ In particular, if we select $\mathcal{Q}_{j}$ to be Hermitian, $F_{j}+K_{j} \mathcal{P}$ will be Hermitian.

It turns out that the condition that $F_{j}+K_{j} \mathcal{P}$ be Hermitian, which can be stated as

$$
\mathcal{P} K_{j}^{\dagger}-K_{j} \mathcal{P}=F_{j}-F_{j}^{\dagger},
$$

is equivalent to the requirement that $F_{j}$ and $K_{j}$ be respectively Hermitian and $\mathcal{P}$-pseudoHermitian [2], i.e.,

$$
F_{j}^{\dagger}=F_{j}, \quad K_{j}^{\dagger}=\mathcal{P} K_{j} \mathcal{P}^{-1}=\mathcal{P} K_{j} \mathcal{P} .
$$

Therefore, as functions of $p, F_{j}$ is real-valued while $K_{j}$ is $\mathcal{P} \mathcal{T}$-invariant. ${ }^{5}$ One way of seeing this is to use the identities $[p, \mathcal{P}]=2 p \mathcal{P}$ and $\left[p, K_{j}\right]=0$ to show that

$$
\left[p, \mathcal{P} K_{j}^{\dagger}-K_{j} \mathcal{P}\right]=2 p\left(\mathcal{P} K_{j}^{\dagger}-K_{j} \mathcal{P}\right) .
$$

In view of (23) and $\left[p, F_{j}\right]=0$, the left-hand side of (25) vanishes identically whereas its right-hand side equals $2 p\left(F_{j}-F_{j}^{\dagger}\right)$. Hence, $F_{j}-F_{j}^{\dagger}=0$, which together with (23) imply (24).

For the case that $H_{0}$ involves a non-vanishing potential $v_{0}$, equations (16) and (19) do not admit a closed form exact solution. In fact, one can prove that the factorization technique that is at the heart of the derivation of d'Alemert's formula (201) does not apply to (19). The spectral method that is also originally developed to solve wave (and heat) equations admit a

\footnotetext{
${ }^{2}$ We will however continue using the label $j$, because for different $j$ we can adopt different homogeneous solutions.

${ }^{3}$ The Hermiticity condition: $f_{j}(x, y)=f_{j}(y, x)^{*}$ imposes the following restrictions on $\varphi_{j}$ and $\chi_{j}: \Re\left[\varphi_{j}(-x)\right]=$ $\Re\left[\varphi_{j}(x)\right], \Im\left[\varphi_{j}(-x)\right]=-\Im\left[\varphi_{j}(x)\right]-2 r_{j}, \Im\left[\chi_{j}(x)\right]=r_{j}$, where $\Re$ and $\Im$ stand for real and imaginary parts of their argument respectively and $r_{j}$ is an arbitrary real constant.

${ }^{4}$ They must also be piece-wise analytic and possess (inverse) Fourier transforms.

${ }^{5}$ This means that for any real variable $p_{c}, F_{j}\left(p_{c}\right) \in \mathbb{R}$ and $K_{j}\left(p_{c}\right)^{*}=K_{j}\left(-p_{c}\right)$.
} 
generalization to both (16) and (19). Yet it does not produce generic closed form expressions for the most general solutions of (16) and (19).

In the remainder of this paper we will only be concerned with the case $v_{0}=0$, i.e., $H_{0}=\frac{1}{2} p^{2}$. In this case we can use the following prescription to compute $\mathcal{Q}_{j}$.

(i) Fourier transform both sides of (17) over $y$ to yield the ordinary differential equation:

$$
\left(\partial_{x}^{2}+p^{2}\right)\left\langle x\left|Q_{j}\right| p\right\rangle=-2\left\langle x\left|R_{j}\right| p\right\rangle
$$

where $p$ is treated as a free real parameter. This equation admits solutions of the form

$$
-2 \int_{x_{0}}^{x} d x^{\prime} G_{p}\left(x, x^{\prime}\right)\left\langle x^{\prime}\left|R_{j}\right| p\right\rangle
$$

where $G_{p}\left(x, x^{\prime}\right)=\sin \left[p\left(x-x^{\prime}\right)\right] / p$ is the Green's function for $\partial_{x}^{2}+p^{2}$;

(ii) Evaluate the integral in (27) by making a simple choice for $x_{0}$ and identify the resulting particular solution of (26) with $\left\langle x\left|\mathcal{Q}_{j}\right| p\right\rangle$.

(iii) Take the inverse Fourier transform of $\left\langle x\left|\mathcal{Q}_{j}\right| p\right\rangle$ over $p$ and denote the result by $\left\langle x\left|\mathcal{Q}_{j}\right| y\right\rangle$. If $\left\langle x\left|\mathcal{Q}_{j}\right| y\right\rangle^{*}=\left\langle y\left|\mathcal{Q}_{j}\right| x\right\rangle$ proceed to $(i v)$. If not, add the homogeneous solutions $u_{ \pm}(p) e^{ \pm i p x}$ to $\left\langle x\left|\mathcal{Q}_{j}\right| p\right\rangle$, redefine $\left\langle x\left|\mathcal{Q}_{j}\right| y\right\rangle$ to be the inverse Fourier transform of $\left\langle x\left|\mathcal{Q}_{j}\right| p\right\rangle+u_{+}(p) e^{i p x}+$ $u_{-}(p) e^{-i p x}$ over $p$, fix $u_{ \pm}$in such a way that $\left\langle x\left|\mathcal{Q}_{j}\right| y\right\rangle^{*}=\left\langle y\left|\mathcal{Q}_{j}\right| x\right\rangle$, and relabel $\left\langle x\left|\mathcal{Q}_{j}\right| p\right\rangle+$ $u_{+}(p) e^{i p x}+u_{-}(p) e^{-i p x}$ by $\left\langle x\left|\mathcal{Q}_{j}\right| p\right\rangle .^{6}$

(iv) Let $\mathcal{Q}_{j}(x, p):=\sqrt{2 \pi} e^{-i x p}\left\langle x\left|\mathcal{Q}_{j}\right| p\right\rangle$ and order the terms in $\mathcal{Q}_{j}(x, p)$ in such a way that all $x$ 's are placed to the left of $p$ 's. This yields $\mathcal{Q}_{j}$, if $x$ and $p$ are identified with the corresponding operators.

This prescription produces an expression for the operator $\mathcal{Q}_{j}$ that is not manifestly Hermitian. To obtain a manifestly Hermitian expression, one must use the standard commutation relations to make the necessary reordering of the terms in $\mathcal{Q}_{j}$. A manifestly Hermitian expression that significantly simplifies the comparison of different operators (or different expressions for the same operator) is the one having the following symmetric form: $g_{0}(x)+h_{0}(p)+\sum_{k=1}^{\infty} s_{k}\left\{g_{k}(x), h_{k}(p)\right\}$, where for all $k \in\{0,1,2, \cdots\}, g_{k}$ and $h_{k}$ are real-valued nonconstant functions, $s_{k} \in \mathbb{R}$, and $\{\cdot, \cdot\}$ is the anticommutator, [6, 7].

\section{$3 \quad$ Metric Operators for $v=i \epsilon x^{3}$}

The imaginary cubic potential, $v(x)=i \epsilon x^{3}$, has a real, positive, discrete spectrum [16, 17, 18, Therefore, according to the general results of [1], it admits a nonempty set of positive-definite metric operators $\eta_{+}$. Because this potential is purely imaginary, it is an ideal toy model to apply the general method developed in the preceding section.

\footnotetext{
${ }^{6}$ One can show that there is always a pair of functions $u_{ \pm}$that satisfy $\left\langle x\left|\mathcal{Q}_{j}\right| y\right\rangle^{*}=\left\langle y\left|\mathcal{Q}_{j}\right| x\right\rangle$.
} 
The calculation of $Q_{j}$ for the potential $v=i \epsilon x^{3}$ has been considered in [14] where the authors obtain the operators $Q_{j}$ that yield the metric operator $\eta_{+}$associated with the $\mathcal{C P} \mathcal{T}$ inner product. They achieve this in two steps: (1) They use the restriction imposed by the choice of the $\mathcal{C P} \mathcal{T}$-inner product to infer that $Q_{j}$ must be even in $x$ and odd in $p$ and to obtain a more restricted set of operator equations for $Q_{j}$ (This amounts to setting $Q_{j}=0$ for even $j$.) (2) They adopt an appropriate ansatz for the form of $Q_{j}$ and determine its free coefficients by enforcing the operator equations for $Q_{j}$.

In this section, we will use our analytic method to obtain the most general $Q_{j}$ for the potential $v=i \epsilon x^{3}$. Our method is more systematic, technically more convenient, and more general, for it does not involve making any kind of (a priori) assumptions about the general structure or symmetries of $Q_{j}$. In particular, it does not rely on making an ansatz for $Q_{j}$. Indeed, we will see that the ansatz used in [14] misses a large class of $\mathcal{C P} \mathcal{T}$-inner products even in the first order of perturbation theory.

As we have argued in the preceding section for purely imaginary potentials such as $v=i \epsilon x^{3}$, the operators $Q_{j}$ have the general form (22). In the following, we will first obtain the structure of the functions $F_{j}$ and $K_{j}$ appearing in (22) and then present our calculation of $\mathcal{Q}_{j}$ and $Q_{j}$ for $j=1,2,3$.

\subsection{Calculation of $F_{j}$ and $K_{j}$}

By definition [4, 19, 5, 6], the physical observables $O$ are linear operators that are Hermitian with respect to the inner product $\langle\cdot, \cdot\rangle_{\eta_{+}}$, i.e., they are $\eta_{+}$-pseudo-Hermitian operators [2]. We can construct them in terms of linear operators $o$ that are Hermitian with respect to the reference inner product $\langle\cdot \mid \cdot\rangle$ according to $O=\eta_{+}^{-1 / 2} o \eta_{+}^{1 / 2}$. For example, for $o=x$ and $o=p$, we find the $\eta_{+}$-pseudo-Hermitian position $(X)$ and momentum $(P)$ operators,

$$
\begin{aligned}
X & :=\eta_{+}^{-1 / 2} x \eta_{+}^{1 / 2}=e^{Q / 2} x e^{-Q / 2}=x+\sum_{k=1}^{\infty} \frac{(-1)^{k}}{2^{k} k !}[x, Q]_{k}, \\
P & :=\eta_{+}^{-1 / 2} p \eta_{+}^{1 / 2}=e^{Q / 2} p e^{-Q / 2}=p+\sum_{k=1}^{\infty} \frac{(-1)^{k}}{2^{k} k !}[p, Q]_{k},
\end{aligned}
$$

respectively, 4, 5]. These equations show how the operators $Q_{j}$ and in particular $F_{j}$ and $K_{j}$ enter the expression for the basic physical observables of the theory.

Next, let $\ell \in \mathbb{R}^{+}$and scale $x$ according to $x \rightarrow x / \ell$. The requirements that the canonical commutation relation is not affected by this scaling and that the Hamiltonian $H=\frac{1}{2} p^{2}+i \epsilon x^{3}$ changes by a total scaling (so that the scaling $x \rightarrow x / \ell$ has the non-physical effect of changing units, as it should), we find that $p \rightarrow \ell p$ and $\epsilon \rightarrow \ell^{5} \epsilon$.

In light of (15), it is not difficult to see that the right-hand side of (28) involves terms of the form $\epsilon^{j}\left[x, F_{j}(p)\right]=i \epsilon^{j} F_{j}^{\prime}(p)$ and $\epsilon^{j}\left[x, K_{j}(p)\right] \mathcal{P}=i \epsilon^{j} K_{j}^{\prime}(p) \mathcal{P}$, where a prime means the first derivative. Therefore, if we demand $X$ to have the same scaling properties as $x$, i.e., $X \rightarrow X / \ell$, we find that $x \rightarrow x / \ell$ implies

$$
F_{j}(p) \rightarrow F_{j}(\ell p)=\ell^{-5 j} F_{j}(p), \quad K_{j}(p) \rightarrow K_{j}(\ell p)=\ell^{-5 j} K_{j}(p) .
$$


In particular, we have $F_{j}(p)=p^{-5 j} \lambda_{j}(p)$ and $K_{j}(p)=p^{-5 j} \theta_{j}(p)$ where $\lambda_{j}(\ell p)=\lambda_{j}(p)$ and $\theta_{j}(\ell p)=\theta_{j}(p)$ for all $\ell \in \mathbb{R}^{+}$. Furthermore, the assumption that the quantum theory under study does not involve a preassigned hidden length scale implies that $\lambda_{j}$ and $\theta_{j}$ must be constants. ${ }^{7}$ This together with the requirement that $F_{j}$ be real-valued and $K_{j}$ be $\mathcal{P} \mathcal{T}$-invariant then imply that $\lambda_{j} \in \mathbb{R}$ and $\theta_{j}=i^{j} \kappa_{j}$ for some $\kappa_{j} \in \mathbb{R}$. Therefore, for the imaginary cubic potential, we have for all $j \in \mathbb{Z}^{+}$:

$$
F_{j}(p)=\frac{\lambda_{j}}{p^{5 j}}, \quad K_{j}(p)=\frac{i^{j} \kappa_{j}}{p^{5 j}}
$$

where $\lambda_{j}, \kappa_{j} \in \mathbb{R}$ are arbitrary. ${ }^{8}$

\subsection{Calculation of $Q_{j}$}

For the potential $v=i \epsilon x^{3}$, we have $H_{0}=\frac{1}{2} p^{2}, H_{1}=i x^{3}$, and $R_{1}=-2 i x^{3}$. Hence, the most general form of $Q_{1}$ is obtained by setting $j=1$ in (22), where $\left\langle x\left|\mathcal{Q}_{1}\right| y\right\rangle$ is a particular solution of the wave equation

$$
\left[-\partial_{x}^{2}+\partial_{y}^{2}\right]\left\langle x\left|Q_{1}\right| y\right\rangle=-4 i x^{3} \delta(x-y)
$$

and $\delta(x)$ denotes the Dirac delta function. To obtain $\mathcal{Q}_{1}$ we use the prescription described in Section 2. Taking the Fourier transform of (32) and solving (26) for a particular solution, we find

$$
\left\langle x\left|\mathcal{Q}_{1}\right| p\right\rangle=\frac{1}{2 \sqrt{2 \pi}} e^{i p x}\left(x^{4} \frac{1}{p}+2 i x^{3} \frac{1}{p^{2}}-3 x^{2} \frac{1}{p^{3}}-3 i x \frac{1}{p^{4}}\right) .
$$

Hence,

$$
\mathcal{Q}_{1}=\frac{1}{2}\left(x^{4} \frac{1}{p}+2 i x^{3} \frac{1}{p^{2}}-3 x^{2} \frac{1}{p^{3}}-3 i x \frac{1}{p^{4}}\right) .
$$

We can evaluate the inverse Fourier transform of (33) to obtain

$$
\left\langle x\left|\mathcal{Q}_{1}\right| y\right\rangle=\frac{i}{8} x y\left(x^{2}+y^{2}\right) \operatorname{sign}(x-y),
$$

where $\operatorname{sign}(x):=x /|x|$ for $x \neq 0$ and $\operatorname{sign}(0):=0$.

Eq. (35) shows that indeed $\mathcal{Q}_{1}$ is a Hermitian operator. With the help of the identities

$$
[x, f(p)]=i f^{\prime}(p), \quad\left[x^{3}, f(p)\right]=\frac{3 i}{2}\left\{x^{2}, f^{\prime}(p)\right\}+\frac{i}{2} f^{(3)}(p),
$$

where $f^{(n)}$ stands for the $n$-th derivative of $f$ and $f^{\prime}:=f^{(1)}$, we can express $\mathcal{Q}_{1}$ in the following manifestly Hermitian form

$$
\mathcal{Q}_{1}=\frac{1}{4}\left\{x^{4}, \frac{1}{p}\right\}+\frac{3}{4}\left\{x^{2}, \frac{1}{p^{3}}\right\}+\frac{3}{p^{5}} .
$$

A useful check on the validity of our calculation of $\mathcal{Q}_{1}$ is to verify that (35) satisfies (32). We have checked this by direct substitution of (35) in the left-hand side of (32).

\footnotetext{
${ }^{7}$ This argument is effectively equivalent to and provides a conceptual interpretation for what the authors' of 14] refer to as "dimensional consistency".

${ }^{8}$ We can also apply the assumption of lack of a hidden length scale in the underlying classical theory to arrive at (31).
} 
Substituting (37) in (22) with $j=1$ and using (31) we find the most general form of $Q_{1}$, namely

$$
Q_{1}=\frac{1}{4}\left\{x^{4}, \frac{1}{p}\right\}+\frac{3}{4}\left\{x^{2}, \frac{1}{p^{3}}\right\}+\frac{\tilde{\lambda}_{1}}{p^{5}}+\frac{i \kappa_{1}}{p^{5}} \mathcal{P},
$$

where $\tilde{\lambda}_{1}:=\lambda_{1}+3$ and $\kappa_{1}$ are arbitrary real constants.

Now, we are in a position to compare our result for $Q_{1}$ with that obtained by Bender, Brody, and Jones (BBJ) [14, namely

$$
Q_{1}^{\mathrm{BBJ}}=\frac{1}{32}\left(x^{4} \frac{1}{p}+4 x^{3} \frac{1}{p} x+6 x^{2} \frac{1}{p} x^{2}+4 x \frac{1}{p} x^{3}+\frac{1}{p} x^{4}\right)+\frac{\alpha}{p},
$$

where $\alpha \in \mathbb{R}$ is arbitrary. We can use the identities,

$$
x^{3} \frac{1}{p} x+x \frac{1}{p} x^{3}=\left\{x^{4}, \frac{1}{p}\right\}+3\left\{x^{2}, \frac{1}{p^{2}}\right\}+\frac{12}{p^{5}}, \quad x^{2} \frac{1}{p} x^{2}=\frac{1}{2}\left\{x^{4}, \frac{1}{p}\right\}+2\left\{x^{2}, \frac{1}{p^{3}}\right\}+\frac{12}{p^{5}},
$$

to express (39) in the form

$$
Q_{1}^{\mathrm{BBJ}}=\frac{1}{4}\left\{x^{4}, \frac{1}{p}\right\}+\frac{3}{4}\left\{x^{2}, \frac{1}{p^{3}}\right\}+\left(\alpha+\frac{15}{4}\right) \frac{1}{p^{5}} .
$$

Clearly, $Q_{1}^{\mathrm{BBJ}}$ defines a one-parameter subfamily of the operators of the form (38). It corresponds to the choice $\tilde{\lambda}_{1}=\alpha+\frac{15}{4}$ and $\kappa_{1}=0$.

It is interesting to see that (38) does also define a $\mathcal{C P} \mathcal{T}$-inner product, for it satisfies all the conditions stated in [14]. In particular, if we use the number operator $N=\frac{1}{2}\left(x^{2}+p^{2}-1\right)$ to express $\mathcal{P}$ in the form ${ }^{9}$

$$
\mathcal{P}=(-1)^{N}=e^{i \pi N}=e^{\frac{i \pi}{2}\left(p^{2}+x^{2}-1\right)}=-i e^{\frac{i \pi}{2}\left(p^{2}+x^{2}\right)}
$$

we see that the last term in (38) is also even in $x$ and odd in $p$. The analysis of [14] seems to have missed this term, because it relies on the choice of a particular ansatz for $Q_{1}$.

Our derivation of $Q_{1}$ shows that, under the assumption that the quantum system defined by the Hamiltonian $H=\frac{p^{2}}{2}+i \epsilon x^{3}$ does not have a hidden length scale, up to (and including) the first order terms in the perturbation parameter $\epsilon$, the $\mathcal{C P} \mathcal{T}$-inner products are the most general inner products that restore the unitarity of the dynamics. This result does not, however, extend to higher orders in perturbation theory. It already fails in the second order, because in general

$$
Q_{2}=F_{2}(p)+K_{2}(p) \mathcal{P}=\frac{1}{p^{10}}\left(\lambda_{2}-\kappa_{2} \mathcal{P}\right),
$$

whereas for a $\mathcal{C P} \mathcal{T}$-inner product $Q_{2}=0$. This is a manifestation of the fact that the $\mathcal{C P} \mathcal{T}$-inner products form a proper subset of the set of all allowed inner products.

Next, we wish to compute $Q_{3}$ using our method. We postpone the details of this calculation to Appendix B. Here we outline its general strategy.

\footnotetext{
${ }^{9}$ Recall that the eigenfunctions $\langle x \mid n\rangle$ of the harmonic oscillator Hamiltonian $\frac{1}{2}\left(x^{2}+p^{2}-1\right)$ are even (respectively odd) functions of $x$ for even (respectively odd) values of the spectral label $n$. Hence $\mathcal{P}=(-1)^{N}$.
} 
The first step in the calculation of $Q_{3}$ is the determination of $R_{3}$ which in view of (13) is given by

$$
R_{3}=-\frac{i}{6}\left[\left[x^{3}, Q_{1}\right], Q_{1}\right]
$$

We recall that according to the prescription explained in Section 2 we need to compute $\left\langle x\left|R_{3}\right| p\right\rangle$. Therefore, instead of evaluating the double commutator in (44), we compute

$$
\left\langle x\left|R_{3}\right| y\right\rangle=-\frac{1}{6}\left\langle x\left|\left[\left[i x^{3}, Q_{1}\right], Q_{1}\right]\right| y\right\rangle=-\frac{i}{6} \int_{-\infty}^{\infty} d z\left(x^{3}+y^{3}-2 z^{3}\right)\left\langle x\left|Q_{1}\right| z\right\rangle\left\langle z\left|Q_{1}\right| y\right\rangle,
$$

and take its Fourier transform over $y .{ }^{10}$ Next, we insert $Q_{1}=\mathcal{Q}_{1}+\lambda p^{-5}+i \kappa p^{-5} \mathcal{P}$, which is equivalent to (38), in Eq. (45) and use (35) to perform the integral over $z$. Doing the necessary calculations, as outlined in Appendix B, we then find

$$
R_{3}=S_{0,0}+\lambda_{1} S_{1,0}+\kappa_{1} S_{0,1}+\lambda_{1} \kappa_{1} S_{1,1}+\lambda_{1}^{2} S_{2,0}+\kappa_{1}^{2} S_{0,2}
$$

where the operators $S_{\mu, \nu}$ are defined by Eqs. (80) - (85) below.

In view of (46), we can construct a particular solution of (17) of the form

$$
\mathcal{Q}_{3}=T_{0,0}+\lambda_{1} T_{1,0}+\kappa_{1} T_{0,1}+\lambda_{1} \kappa_{1} T_{1,1}+\lambda_{1}^{2} T_{2,0}+\kappa_{1}^{2} T_{0,2}
$$

where $T_{\mu, \nu}$ solves the operator equation

$$
\left[p^{2}, T_{\mu, \nu}\right]=2 S_{\mu, \nu}
$$

Hence, $\left\langle x\left|T_{\mu, \nu}\right| y\right\rangle$ is a particular solution of the wave equation

$$
\left(-\partial_{x}^{2}+\partial_{y}^{2}\right)\left\langle x\left|T_{\mu, \nu}\right| y\right\rangle=2\left\langle x\left|S_{\mu, \nu}\right| y\right\rangle
$$

In Appendix B we construct such solutions and check that the corresponding operators, $T_{\mu, \nu}$, are Hermitian. This shows that the operator $\mathcal{Q}_{3}$, as given by (47), is Hermitian. Consequently Eqs. (31) hold for $j=3$.

Using (22), (31), (47), and the explicit form of the operators $T_{\mu, \nu}$ given in Appendix B, we obtain after a lengthy calculation

$$
Q_{3}=\sum_{\ell=1}^{5} d_{0 \ell}\left\{x^{2 \ell}, \frac{1}{p^{15-2 \ell}}\right\}+\frac{\tilde{\lambda}_{3}}{p^{15}}-i\left(\sum_{\ell=1}^{4} d_{1 \ell}\left\{x^{2 \ell}, \frac{1}{p^{15-2 \ell}}\right\}+\frac{\tilde{\kappa}_{3}}{p^{15}}\right) \mathcal{P}
$$

where

$$
\begin{aligned}
d_{01} & :=c_{001}+\lambda_{1} c_{101}+\lambda_{1}^{2} c_{201}+\kappa_{1}^{2} c_{021}, & d_{02}:=c_{002}+\lambda_{1} c_{102}+\kappa_{1} c_{012} \\
d_{03}:=c_{003}+\lambda_{1} c_{103}, & d_{04}=c_{004}, & d_{05}=c_{005} \quad d_{11}:=\kappa_{1}\left(c_{011}+\lambda_{1} c_{111}\right), \\
d_{12}:=\kappa_{1}\left(c_{012}+\lambda_{1} c_{112}\right), & d_{13}:=\kappa_{1} c_{013}, & d_{14}:=\kappa_{1} c_{014}, \\
\tilde{\lambda}_{3}:= & \lambda_{3}+2\left(c_{000}+\lambda_{1} c_{100}+\lambda_{1}^{2} c_{200}+\kappa_{1}^{2} c_{020}\right), & \tilde{\kappa}_{3}:=\kappa_{3}+2 \kappa_{1}\left(c_{010}+\lambda_{1} c_{110}\right),
\end{aligned}
$$

\footnotetext{
${ }^{10}$ The advantage of this approach is that the above calculation can be done using Mathematica.
} 
$c_{\mu \nu \ell}$ are real constants given in Table 1, $\lambda_{1}, \kappa_{1}$ are the free real parameters determining $Q_{1}$, and $\lambda_{3}, \kappa_{3}$ (or equivalently $\tilde{\lambda}_{3}, \tilde{\kappa}_{3}$ ) are another pair of free real parameters.

Note that the freedom in the choice of parameters $\alpha$ and $\beta$ entering the expression obtained in [14] for $Q_{3}$ corresponds to the freedom in the choice of $\lambda_{1}$ and $\lambda_{3}$ (or $\tilde{\lambda}_{3}$ ). Furthermore, it is not difficult to see that $Q_{3}$ constructed above fulfils all the conditions imposed by the choice of $\mathcal{C P} \mathcal{T}$-inner product. The fact that the approach of [14] do not reveal the presence of the terms proportional to $\kappa_{1}$ and $\tilde{\kappa}_{3}$ stems from the particular choice for the ansatz used in [14].

We conclude this section by mentioning that we can similarly apply our method to obtain the general form of $Q_{j}$ for $j>3$. But as expected the algebra becomes quite involved.

\section{Physical Implications of Changing the Metric Opera- tor in Quantum and Classical Treatments}

In order to understand how the freedom in the choice of the metric operator affects the physical content of the theory, we will use the most general metric operator to perform a perturbative calculation of the pseudo-Hermitian position (28) and momentum (29) operators, the equivalent Hermitian Hamiltonian,

$$
h:=\eta_{+}^{1 / 2} H \eta_{+}^{-1 / 2}=e^{-Q / 2} H e^{Q / 2},
$$

and the classical Hamiltonian ${ }^{11}$,

$$
H_{c}\left(x_{c}, p_{c}\right):=\left.\lim _{\hbar \rightarrow 0} h(x, p)\right|_{x \rightarrow x_{c}, p \rightarrow p_{c}},
$$

for the system defined by the imaginary cubic potential.

We can easily show, using (6), (28), (29) and (51), that

$$
\begin{aligned}
X & =x-\frac{1}{2}\left[x, Q_{1}\right] \epsilon+\mathcal{O}\left(\epsilon^{2}\right), \quad P=p-\frac{1}{2}\left[p, Q_{1}\right] \epsilon+\mathcal{O}\left(\epsilon^{2}\right), \\
h & =\frac{p^{2}}{2}+\frac{i}{4}\left[x^{3}, Q_{1}\right] \epsilon^{2}++\frac{i}{4}\left[x^{3}, Q_{2}\right] \epsilon^{3}+\mathcal{O}\left(\epsilon^{4}\right),
\end{aligned}
$$

where $\mathcal{O}\left(\epsilon^{n}\right)$ stand for terms of order $n$ and higher in powers of $\epsilon$. Therefore, we first use (38) and (43) to compute

$$
\begin{aligned}
& {\left[x, Q_{1}\right]=-\frac{i}{4}\left(\left\{x^{4}, \frac{1}{p^{2}}\right\}+9\left\{x^{2}, \frac{1}{p^{4}}\right\}+\frac{20 \tilde{\lambda}_{1}}{p^{6}}-4 \kappa_{1}\left\{x, \frac{1}{p^{5}}\right\} \mathcal{P}\right)} \\
& {\left[p, Q_{1}\right]=-\frac{i}{2}\left(2\left\{x^{3}, \frac{1}{p}\right\}+3\left\{x, \frac{1}{p^{3}}\right\}-\frac{4 \kappa_{1}}{p^{4}} \mathcal{P}\right)} \\
& {\left[x^{3}, Q_{1}\right]=-\frac{3 i}{4}\left(\left\{x^{6}, \frac{1}{p^{2}}\right\}+22\left\{x^{4}, \frac{1}{p^{4}}\right\}+\left(510+10 \tilde{\lambda}_{1}\right)\left\{x^{2}, \frac{1}{p^{6}}\right\}+\right.} \\
& \left.\frac{8820+140 \tilde{\lambda}_{1}}{p^{8}}-\frac{4}{3} \kappa_{1}\left\{x^{3}, \frac{1}{p^{5}}\right\} \mathcal{P}\right) \\
& {\left[x^{3}, Q_{2}\right]=-15 i \lambda_{2}\left(\left\{x^{2}, \frac{1}{p^{11}}\right\}+\frac{44}{p^{13}}\right)-\kappa_{2}\left\{x^{3}, \frac{1}{p^{10}}\right\} \mathcal{P} .}
\end{aligned}
$$

\footnotetext{
${ }^{11} x_{c}$ and $p_{c}$ appearing in (52) are respectively the classical position and momentum observables.
} 
Substituting these relations in (153) and (154), we have

$$
\begin{aligned}
& X= x+\frac{i}{8}\left(\left\{x^{4}, \frac{1}{p^{2}}\right\}+9\left\{x^{2}, \frac{1}{p^{4}}\right\}+\frac{20 \tilde{\lambda}_{1}}{p^{6}}-4 \kappa_{1}\left\{x, \frac{1}{p^{5}}\right\} \mathcal{P}\right) \epsilon+\mathcal{O}\left(\epsilon^{2}\right), \\
& P= p+\frac{i}{4}\left(2\left\{x^{3}, \frac{1}{p}\right\}+3\left\{x, \frac{1}{p^{3}}\right\}-\frac{4 \kappa_{1}}{p^{4}} \mathcal{P}\right) \epsilon+\mathcal{O}\left(\epsilon^{2}\right), \\
& h= \frac{p^{2}}{2}+\frac{3}{16}\left(\left\{x^{6}, \frac{1}{p^{2}}\right\}+22\left\{x^{4}, \frac{1}{p^{4}}\right\}+\left(510+10 \tilde{\lambda}_{1}\right)\left\{x^{2}, \frac{1}{p^{6}}\right\}+\right. \\
&\left.\frac{8820+140 \tilde{\lambda}_{1}}{p^{8}}-\frac{4}{3} \kappa_{1}\left\{x^{3}, \frac{1}{p^{5}}\right\} \mathcal{P}\right) \epsilon^{2}+ \\
& \frac{1}{4}\left(15 \lambda_{2}\left(\left\{x^{2}, \frac{1}{p^{11}}\right\}+\frac{44}{p^{13}}\right)-i \kappa_{2}\left\{x^{3}, \frac{1}{p^{10}}\right\} \mathcal{P}\right) \epsilon^{3}+\mathcal{O}\left(\epsilon^{4}\right) .
\end{aligned}
$$

Equations (59) and (60) show how the free parameters $\tilde{\lambda}_{1}$ and $\kappa_{1}$, that determine the metric operator up to terms of order $\epsilon$, enter the definition of the basic observables of the theory.

The quantum theory defined by the imaginary cubic potential can be described by the manifestly Hermitian Hamiltonian $h$ within the framework of the standard quantum mechanics, [20, 19]. However, as seen from Eq. (61) this equivalent Hermitian description is sensitive to the choice of the metric. The metric-independence of the Hamiltonian $H$ and the metric-dependence of the physical observables $O$ in the non-Hermitian description of the quantum system are traded with the metric-dependence of the Hamiltonian $h$ and the metric-independence of the physical observables o in its Hermitian description.

Having calculated the Hermitian Hamiltonian $h$, we can determine the classical Hamiltonian (52). This requires making the $\hbar$-dependence of the terms in (61) explicit. We do this by letting $h \rightarrow \ell^{-2} m^{-1} \hbar^{2} h, x \rightarrow \ell^{-1} x, p \rightarrow \ell \hbar^{-1} p$, where $\ell$ is an arbitrary length scale and $m$ is the mass, 6]. Making this transformations, replacing the quantum observables with their classical counterparts, i.e., $x \rightarrow x_{c}$ and $p \rightarrow p_{c}$, and taking the limit $\hbar \rightarrow 0$, we find the following remarkably simple expression for the classical Hamiltonian (52).

$$
H_{c}=\frac{p_{c}^{2}}{2 m}+\frac{3}{8} m \epsilon^{2} \frac{x_{c}^{6}}{p_{c}^{2}}+\mathcal{O}\left(\epsilon^{4}\right) .
$$

Clearly $H_{c}$ is an even and nonnegative function of $x_{c}$ and $p_{c}$. This is an indication that it supports closed classical phase space orbits. As shown in Figure 1, this is actually the case.

A more important observation is that the terms in (61) that involve $\tilde{\lambda}_{1}, \kappa_{1}, \lambda_{2}$ and $\kappa_{2}$ do not contributes to the classical Hamiltonian, because they involve positive integer powers of $\hbar$ that vanish in the classical limit $\hbar \rightarrow 0$. It is not difficult to see that the same behavior holds in all orders of perturbation; the terms involving $\lambda_{j}$ and $\kappa_{j}$, that characterize the freedom in the choice of the metric operator, do not contribute to the classical Hamiltonian. This means, at least for the system we consider, that the classical Hamiltonian is not sensitive to the choice of the metric operator. We expect this assertion to hold true generally. 


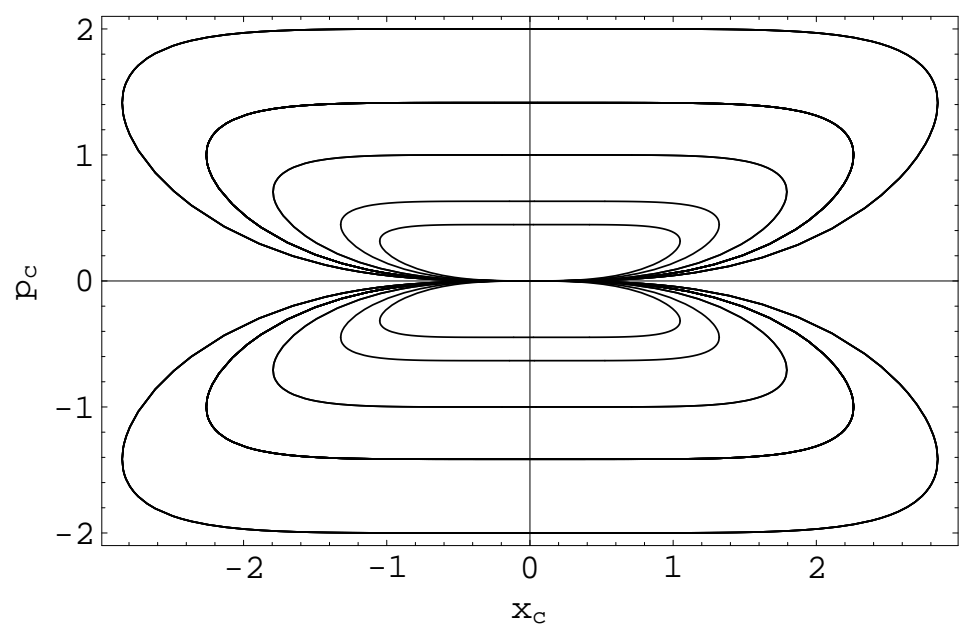

Figure 1: Typical phase-space orbits for the classical Hamiltonian $H_{c}$, for $\epsilon=0.1$ and $m=1$.

\section{Pseudo-Hermitian Quantization of the Free Particle}

If we set $H_{0}=p^{2} / 2$ and $v_{0}=H_{1}=0$ in the constructions of Section 2, we find the most general metric operator $\eta_{+}=e^{-Q}$ that renders the free particle Hamiltonian $H=H_{0}$ Hermitian. It is not difficult to see that in this case $R_{j}=0$ for all $j \in \mathbb{Z}^{+}$and that

$$
Q=F+K \mathcal{P}
$$

for a pair of functions $F$ and $K$ of $p$. Again the fact that the system does not involve a particular length scale implies that $F(p)=: \lambda$ and $K(p)=: \kappa$ are real constants. Hence

$$
\eta_{+}=e^{-\lambda} e^{-\kappa \mathcal{P}}=e^{-\lambda}[\cosh (\kappa)-\sinh (\kappa) \mathcal{P}] .
$$

For this system the pseudo-Hermitian position (28) and momentum (29) operators take the form

$$
X=x e^{-\kappa \mathcal{P}}=e^{\kappa \mathcal{P}} x, \quad P=p e^{-\kappa \mathcal{P}}=e^{\kappa \mathcal{P}} p .
$$

Clearly the Hermitian hamiltonian $h$ and the classical Hamiltonian $H_{c}$ coincide with $H$ and $p_{c}^{2} / 2$, respectively. The pseudo-Hermitian quantization scheme defined by

$$
x_{c} \rightarrow X \quad p_{c} \rightarrow P, \quad \text { Poission bracket } \rightarrow-i \text { commutator }
$$

is the most general physically admissible canonical quantization of the free particle in one dimension that is compatible with the usual form of the free particle Hamiltonian. Note that for $\kappa \neq 0$ the operators $X$ and $P$ are not Hermitian with respect to the usual $L^{2}$-inner product. Yet they furnish a unitary irreducible representation of the Heisenberg-Weyl algebra $[x, p]=i$. The representation space is the Hilbert space $\mathcal{H}_{\text {phys }}=\left\{\psi: \mathbb{R} \rightarrow \mathbb{C} \mid\langle\psi, \psi\rangle_{\eta_{+}}<\infty\right\}$ endowed with the inner product,

$$
\langle\phi, \psi\rangle_{\eta_{+}}:=\left\langle\phi\left|\eta_{+}\right| \psi\right\rangle=e^{-\lambda / 2} \cosh (\kappa) \int_{-\infty}^{\infty} d x \phi(x)^{*} \psi(x)-e^{-\lambda / 2} \sinh (\kappa) \int_{-\infty}^{\infty} d x \phi(x)^{*} \psi(-x),
$$


where $\psi, \phi: \mathbb{R} \rightarrow \mathbb{C}$ are arbitrary functions. ${ }^{12}$

As suggested by (65) and (67), the pseudo-Hermitian quantization of the free particle may be viewed as a smooth deformation of the standard (Hermitian) canonical quantization (which corresponds to taking $\kappa=0$ ).

Next, we recall that $\eta_{+}^{1 / 2}$ defines a unitary operator mapping $\mathcal{H}_{\text {phys }}$ onto $L^{2}(\mathbb{R})$, [20, [5] 6]. Under this mapping $X$ and $P$ are transformed to $x$ and $p$ respectively and $H$ is left invariant. Therefore, the pseudo-Hermitian quantum systems associated with all $\lambda, \kappa \in \mathbb{R}$ are physically equivalent. In particular, there is no physical reason to choose $\kappa=0$. We should like to warn however that taking $\kappa \neq 0$ has unusual yet completely consistent consequences. For example, the localized states are described by wave functions that in general have two delta function singularities! Up to an irrelevant phase factor, the delta-function normalized localized state vector centered at $y \in \mathbb{R}$ is given by $\left|\xi^{(y)}\right\rangle:=\eta_{+}^{-1 / 2}|y\rangle$, [5, 6]. Therefore, according to (64),

$$
\left\langle x \mid \xi^{(y)}\right\rangle=e^{\lambda / 2}\left[\cosh \left(\frac{\kappa}{2}\right) \delta(x-y)+\sinh \left(\frac{\kappa}{2}\right) \delta(x+y)\right]
$$

Note however that this is not the physical position wave function for the localized state. The latter has the form $\left\langle\xi^{(x)}, \xi^{(y)}\right\rangle_{\eta_{+}}=\langle x \mid y\rangle=\delta(x-y)$.

Finally, we would like to point out that according to (65), $X^{2}=x^{2}, X P=x p, P^{2}=p^{2}$. Therefore if we perform the pseudo-Hermitian canonical quantization (66) on any classical Hamiltonian $H_{c}$ that can be constructed out of $x_{c}^{2}, x_{c} p_{c}$ and $p_{c}^{2}$, we obtain the same expression for the quantum Hamiltonian as the one obtained using the usual canonical quantization. Conversely, every quantum Hamiltonian $H$ that can be expressed in terms of $x^{2}, x p+p x$, and $p^{2}$ admits a metric operator of the form (64).

\section{Higher Dimensions and Scaling-Invariance}

The situation is considerably more complicated in $n$-dimensional configuration spaces with $n>1$. In this case the operator equations (77) correspond to certain ultrahyperbolic equations in $(n+n)$-dimensions. These equations have peculiar properties, and except for very special cases their standard Cauchy problem is not well-posed [21]. For the free particle in $n$-dimensions, $Q$ satisfies

$$
\left(-\nabla_{x}^{2}+\nabla_{y}^{2}\right)\langle\vec{x}|Q| \vec{y}\rangle=0,
$$

where $\vec{x}=\left(x_{1}, x_{2}, \cdots, x_{n}\right)$ and $\nabla_{x}^{2}:=\sum_{i=1}^{n} \partial_{x_{i}}^{2}$. It is not difficult to see that (63) does not provide the most general solution of (68) $\cdot{ }^{13}$ It only provides a special class of solutions. An important distinction between this class for $n>1$ and the corresponding general solution for $n=1$ is that for $n>1$ the requirement that the theory must not depend on an arbitrary length scale does not restrict $F$ and $K$ to constants. This is simply because one can now use the ratios

\footnotetext{
${ }^{12}$ Because the metric operator (64) is a bounded operator acting in $L^{2}(\mathbb{R}), \mathcal{H}_{\text {phys }}$ and $L^{2}(\mathbb{R})$ are identical as vector spaces. What distinguishes them from one another is their inner products.

${ }^{13}$ For example, for $n=2$, the angular momentum operator, $Q=x_{1} p_{2}-x_{2} p_{1}$, which is clearly not of the form (63), provides a solution of (68).
} 
of the components $p_{i}$ of the momentum operator $\vec{p}$ to construct scaling-independent operators, i.e., require that $F$ and $K$ are functions of the homogeneous $p$-coordinates. For example for $n=2$, we can take $F=F\left(p_{1} / p_{2}\right)$ and $K=K\left(p_{1} / p_{2}\right)$.

A similar situation occurs in one-dimension provided that the system involves an $\epsilon$-independent length scale. A simple example is the scattering potential,

$$
v(x)=\left\{\begin{array}{ccc}
-i \epsilon \operatorname{sign}(x) & \text { for } & |x|<\frac{L}{2} \\
0 & \text { for } & |x| \geq \frac{L}{2}
\end{array}\right.
$$

which includes the length scale $L$. This potential has a real spectrum [7]. It is also imaginary. Therefore, we can apply our method to obtain the general form of $Q_{j}$. In [7] we have used a considerably more difficult spectral method to obtain the following particular solution of (17)).

$$
\mathcal{Q}_{1}(x, y)=\left\langle x\left|\mathcal{Q}_{1}\right| y\right\rangle=\frac{i L}{16}(|x+y+L|+|x+y-L|-2|x+y|-2 L) \operatorname{sign}(x-y) .
$$

According to the results of Section 2, the general form of $Q_{1}$ is given by (22) with $j=1$. However, unlike the case of imaginary cubic potential, the operators $F_{j}$ and $K_{j}$ appearing in (22) can now be complicated functions of $L p$. Specifically, given the fact that for the system defined by the potential (69), the scaling transformation $x \rightarrow x / \ell$ induces the transformation $\epsilon \rightarrow \ell^{2} \epsilon$, it is not difficult to infer that $F_{j}$ and $K_{j}$ have the form

$$
F_{j}=\frac{\lambda_{j}(L p)}{p^{2 j}}, \quad K_{j}=\frac{\kappa_{j}(L p)}{p^{2 j}},
$$

where $\lambda_{j}$ and $\kappa_{j}$ are respectively real-valued and $\mathcal{P} \mathcal{T}$-invariant functions. In particular, we have

$$
Q_{1}=\mathcal{Q}_{1}+p^{-2}\left[\lambda_{1}(L p)+\kappa_{1}(L p) \mathcal{P}\right]
$$

where $\mathcal{Q}_{1}$ is given by $(170)$ and

$$
Q_{2}=p^{-4}\left[\lambda_{2}(L p)+\kappa_{2}(L p) \mathcal{P}\right]
$$

The derivation of (172) using the spectral approach pursued in [7] is an extremely difficult (and open) problem.

\section{Concluding Remarks}

In this paper we offer a method to compute the most general metric operator for a given quasiHermitian standard Hamiltonian in one-dimension. This method can be conveniently used for imaginary potentials. It allows a better understanding of the issue of non-uniqueness of the metric operator and the positive-definite inner products that render the quantum theory unitary.

Applying this method to the imaginary cubic potential we discovered a new class of $\mathcal{C P} \mathcal{T}$ inner products and established the existence of admissible non- $\mathcal{C P} \mathcal{T}$-inner products. Furthermore, we showed that the freedom in the choice of the metric operator affected the basic physical 
observables of the theory in its non-Hermitian representation and the Hamiltonian in its Hermitian representation. An interesting consequence of our investigation is that the underlying classical system is not sensitive to the choice of the inner product.

We also used our method to obtain a complete characterization of pseudo-Hermitian quantization of the free particle Hamiltonian in one-dimension.

We wish to emphasize that the choice of the metric operator can be related to that of an irreducible set of observables as explained in [22. Specifically, the requirement that the members of an irreducible set of operators ${ }^{14}$ be Hermitian fixes the metric operator (up to an irrelevant constant coefficient) [22, 23]. The application of this program for $\mathcal{P} \mathcal{T}$-symmetric Hamiltonians having a real spectrum involves selecting sufficiently many operators (all having real spectra) such that together with the Hamitonian they form an irreducible set. These operators must however be compatible in the sense that there must exist a positive-definite metric operator $\eta_{+}$such that all of them be $\eta_{+}$-pseudo-Hermitian. In general the determination of the compatibility of the operators that are to be chosen is a difficult task. In particular it requires the knowledge of the most general positive-definite metric operator that renders the Hamiltonian pseudo-Hermitian. Therefore, the results we have reported in this paper play a central role in employing the method of 22 in $\mathcal{P} \mathcal{T}$-symmetric quantum mechanics.

End Note: For the imaginary cubic potential considered here it is sometimes argued that $\langle x|\eta| y\rangle$ is not an analytic function of $\epsilon$ and its series expansion studied in [14 and here is meaningless, because $\epsilon$ is not dimensionless and scaling $x$ and $y$ one can make the numerical value of $\epsilon$ as large or small as one wishes. This argument is inconclusive, for it certainly applies to $e^{\epsilon x^{5}}$, which is an analytic function of $\epsilon$ for all $x \in \mathbb{R}$, and to $\left(1+\epsilon x^{5}\right)^{-1}$ which is analytic in $\epsilon$ for $|x|<|\epsilon|^{1 / 5}$. As these examples suggest, in general there is a region in the $x-y$ plane in which $\langle x|\eta| y\rangle$ admits a power series expansion in $\epsilon$. The issue of the summability of this series is an extremely difficult open problem. The fact that $\epsilon$ is not dimensionless implies that an approximate evaluation of $\eta$ that is based on a truncation of the power series for $\langle x|\eta| y\rangle$ will be valid only within a region $\mathcal{D}_{\epsilon}$ of the $x-y$ plane whose size depends on $\epsilon$. Obviously, because the contribution of every order in $\epsilon$ to $\eta$ is dimensionless, scaling $\epsilon$ would scale the size of $\mathcal{D}_{\epsilon}$.

\footnotetext{
${ }^{14} \mathrm{~A}$ set of operators acting in a Hilbert space $\mathcal{H}$ is called irreducible if there is no proper subspace of $\mathcal{H}$ that is left invariant under the action of all members of this set.
} 


\section{Appendix A: Derivation of the Operator Equations Sat- isfied by $Q_{j}$}

In order to derive (7) we first substitute (3) and (41) in (21) and use (6) and the fact that $H_{1}^{\dagger}=-H_{1}$ to obtain

$$
\epsilon H_{1}=-\frac{1}{2}\left(\llbracket H_{0}, Q \rrbracket_{1}+\llbracket \epsilon H_{1}, Q \rrbracket_{1}\right)
$$

where

$$
\llbracket A, Q \rrbracket_{1}:=e^{-Q} A e^{Q}-A=\sum_{k=1}^{\infty} \frac{1}{k !}[A, Q]_{k} .
$$

Next, we substitute the right-hand side of Eq. (173) for the $\epsilon H_{1}$ in the term $\llbracket \epsilon H_{1}, Q \rrbracket_{1}$ that appears in this equation and do the same in the right-hand side of the resulting equation. Repeating this procedure $\ell-1$ times yields

$$
\epsilon H_{1}=\frac{1}{2} \sum_{m=1}^{\ell} \sum_{k=m}^{\infty} q_{m k}\left[H_{0}, Q\right]_{k}+\frac{(-1)^{\ell}}{2^{\ell}} \llbracket \epsilon H_{1}, Q \rrbracket_{\ell}
$$

where $\ell \in \mathbb{Z}^{+}$is arbitrary and for all $k, m \in \mathbb{Z}^{+}$

$$
q_{m k}:=\sum_{n=1}^{m} \frac{(-1)^{n} n^{k}}{k ! 2^{m-1}}\left(\begin{array}{c}
m \\
n
\end{array}\right), \quad\left(\begin{array}{c}
m \\
n
\end{array}\right):=\frac{m !}{n !(m-n) !}, \quad \llbracket A, Q \rrbracket_{k+1}:=\llbracket \llbracket A, Q \rrbracket_{k}, Q \rrbracket_{1} .
$$

In view of (15), (74), (75), and (76), it is easy to see that

$$
\epsilon H_{1}=\frac{1}{2} \sum_{m=1}^{\ell} \sum_{k=m}^{\ell} q_{m k}\left[H_{0}, Q\right]_{k}+\mathcal{O}\left(\epsilon^{\ell+1}\right)
$$

where $\mathcal{O}\left(\epsilon^{\ell}\right)$ stands for terms of order $\ell$ or higher in powers of $\epsilon$. We can employ the identity $\sum_{m=1}^{\ell} \sum_{k=m}^{\ell}=\sum_{k=1}^{\ell} \sum_{m=1}^{k}$ to express (77) in the form

$$
\epsilon H_{1}=\frac{1}{2} \sum_{k=1}^{\ell} q_{k}\left[H_{0}, Q\right]_{k}+\mathcal{O}\left(\epsilon^{\ell+1}\right)
$$

where $q_{k}:=\sum_{m=1}^{k} q_{m k}$.

Next, we substitute (5) in(78) and require that (178) be satisfied at each order $j \leq \ell$ of the perturbation. For $j=1$, this implies

$$
H_{1}=-\frac{1}{2}\left[H_{0}, Q_{1}\right]
$$

where we have used $q_{1}=-1$. For $j \geq 2$, we solve for $\epsilon^{j}\left[H_{0}, Q_{j}\right]$ in terms of the remaining terms of order $\epsilon^{j}$ in (178). This together with (79) yield (7). 


\section{Appendix B: Calculation of $Q_{3}$}

In view of (45), the operator $S_{\mu, \nu}$, that determine $R_{3}$ according to (46), are given by

$$
\begin{aligned}
\left\langle x\left|S_{0,0}\right| y\right\rangle & :=-\frac{i}{6} \int_{-\infty}^{\infty} d z\left(x^{3}+y^{3}-2 z^{3}\right)\left\langle x\left|\mathcal{Q}_{1}\right| z\right\rangle\left\langle z\left|\mathcal{Q}_{1}\right| y\right\rangle \\
\left\langle x\left|S_{1,0}\right| y\right\rangle & :=-\frac{i}{6} \int_{-\infty}^{\infty} d z\left(x^{3}+y^{3}-2 z^{3}\right)\left[\left\langle x\left|\mathcal{Q}_{1}\right| z\right\rangle\left\langle z\left|p^{-5}\right| y\right\rangle+\left\langle x\left|p^{-5}\right| z\right\rangle\left\langle z\left|\mathcal{Q}_{1}\right| y\right\rangle\right] \\
\left\langle x\left|S_{0,1}\right| y\right\rangle & :=\frac{1}{6} \int_{-\infty}^{\infty} d z\left(x^{3}+y^{3}-2 z^{3}\right)\left[\left\langle x\left|\mathcal{Q}_{1}\right| z\right\rangle\left\langle z\left|p^{-5}\right|-y\right\rangle+\left\langle x\left|p^{-5}\right|-z\right\rangle\left\langle z\left|\mathcal{Q}_{1}\right| y\right\rangle\right] \\
\left\langle x\left|S_{1,1}\right| y\right\rangle & :=\frac{1}{6} \int_{-\infty}^{\infty} d z\left(x^{3}+y^{3}-2 z^{3}\right)\left[\left\langle x\left|p^{-5}\right| z\right\rangle\left\langle z\left|p^{-5}\right|-y\right\rangle+\left\langle x\left|p^{-5}\right|-z\right\rangle\left\langle z\left|p^{-5}\right| y\right\rangle\right], \\
\left\langle x\left|S_{2,0}\right| y\right\rangle & :=-\frac{i}{6} \int_{-\infty}^{\infty} d z\left(x^{3}+y^{3}-2 z^{3}\right)\left\langle x\left|p^{-5}\right| z\right\rangle\left\langle z\left|p^{-5}\right| y\right\rangle \\
\left\langle x\left|S_{0,2}\right| y\right\rangle & :=-\frac{1}{6} \int_{-\infty}^{\infty} d z\left(x^{3}+y^{3}-2 z^{3}\right)\left\langle x\left|p^{-5}\right|-z\right\rangle\left\langle z\left|p^{-5}\right|-y\right\rangle
\end{aligned}
$$

We can use (35) and the identities

$$
\operatorname{sign}(x)=\frac{1}{i \pi} \int_{-\infty}^{\infty} d k \frac{e^{i x k}}{k}, \quad\left\langle x\left|\frac{1}{p^{5}}\right| y\right\rangle=\frac{1}{2 \pi} \int_{-\infty}^{\infty} d k \frac{e^{i(x-y) k}}{k^{5}}=\frac{i}{48}(x-y)^{4} \operatorname{sign}(x-y),
$$

to evaluate the integrals appearing in (80) - (85) by turning them into a pair of Fourier transforms that we can easily perform using Mathematica. The result is

$$
\begin{aligned}
\left\langle x\left|S_{0,0}\right| y\right\rangle & =-\frac{i}{26880}\left[15\left(x^{11} y-x y^{11}\right)+7\left(x^{9} y^{3}-x^{3} y^{9}\right)-48\left(x^{8} y^{4}-x^{4} y^{8}\right)\right] \operatorname{sign}(x-y), \\
\left\langle x\left|S_{0,1}\right| y\right\rangle= & \frac{1}{319334400}(x+y)^{5}\left[3115\left(x^{7}-y^{7}\right)-3818\left(x^{6} y-x y^{6}\right)+\right. \\
\left\langle x\left|S_{1,0}\right| y\right\rangle= & -\frac{i}{6386688}(x-y)^{7}\left[623\left(x^{5} y^{2}-x^{2} y^{5}\right)-12123\left(x^{4} y^{3}-x^{3} y^{4}\right)\right] \operatorname{sign}(x+y), 1970\left(x^{4} y+x y^{4}\right)+ \\
\left\langle x\left|S_{1,1}\right| y\right\rangle= & \frac{1}{47900160}(x+y)^{9}\left[7\left(x^{3}-y^{3}\right)-15\left(x^{2} y-x y^{2}\right)\right] \operatorname{sign}(x+y), \\
\left\langle x\left|S_{0,2}\right| y\right\rangle= & \frac{i}{95800320}(x-y)^{9}\left[29\left(x^{3}+y^{3}\right)+15\left(x^{2} y+x y^{2}\right)\right] \operatorname{sign}(x-y), \\
\left\langle x\left|S_{2,0}\right| y\right\rangle= & \frac{i}{6386688}(x-y)^{11}(x+y) \operatorname{sign}(x-y) .
\end{aligned}
$$

These equations show that, as expected, $S_{\mu, \nu}$ are anti-Hermitian.

Next, we use (87) - (92) and the the prescription given in Section 2 to transform the wave equations (49) into the ordinary differential equation $\left(\partial_{x}^{2}+p^{2}\right)\left\langle x\left|T_{\mu, \nu}\right| p\right\rangle=-2\left\langle x\left|S_{\mu, \nu}\right| p\right\rangle$, obtain particular solutions of this equation, and determine the corresponding operators $T_{\mu, \nu}$ that solve 
(48). This yields

$$
\begin{array}{ll}
T_{0,0}=-\sum_{\ell=1}^{10}(-i)^{\ell} a_{00 \ell} x^{\ell} \frac{1}{p^{15-\ell}}, & T_{0,1}=\sum_{\ell=1}^{8}(-i)^{\ell+1} a_{01 \ell} x^{\ell} \frac{1}{p^{15-\ell}} \mathcal{P}, \\
T_{1,0}=-\sum_{\ell=1}^{6}(-i)^{\ell} a_{10 \ell} x^{\ell} \frac{1}{p^{15-\ell}}, & T_{1,1}=\sum_{\ell=1}^{4}(-i)^{\ell+1} a_{11 \ell} x^{\ell} \frac{1}{p^{15-\ell}} \mathcal{P}, \\
T_{0,2}=\sum_{\ell=1}^{4}(-i)^{\ell} a_{02 \ell} x^{\ell} \frac{1}{p^{15-\ell}}, & T_{2,0}=\sum_{\ell=1}^{2}(-i)^{\ell} a_{20 \ell} x^{\ell} \frac{1}{p^{15-\ell}},
\end{array}
$$

where $a_{\mu \nu \ell}$ are real and positive constants given in Table 2 ,

Next, we wish to determine if $T_{\mu, \nu}$ and consequently the operator $\mathcal{Q}_{3}$ given by (47) are Hermitian. We can easily answer this question by inspecting the matrix elements:

$$
\begin{aligned}
\left\langle x\left|T_{0,0}\right| y\right\rangle & =i(x-y)^{4} \sum_{\ell=1}^{5} b_{00 \ell}\left(x^{\ell} y^{10-\ell}+x^{10-\ell} y^{\ell}\right) \operatorname{sign}(x-y), \\
\left\langle x\left|T_{0,1}\right| p\right\rangle & =(x+y)^{6} \sum_{\ell=1}^{4} b_{01 \ell}\left(x^{\ell} y^{8-\ell}+x^{8-\ell} y^{\ell}\right) \operatorname{sign}(x+y), \\
\left\langle x\left|T_{1,0}\right| y\right\rangle & =i(x-y)^{8} \sum_{\ell=1}^{3} b_{10 \ell}\left(x^{\ell} y^{6-\ell}+x^{6-\ell} y^{\ell}\right) \operatorname{sign}(x-y), \\
\left\langle x\left|T_{1,1}\right| y\right\rangle & =(x+y)^{10} \sum_{\ell=1}^{2} b_{11 \ell}\left(x^{\ell} y^{4-\ell}+x^{4-\ell} y^{\ell}\right) \operatorname{sign}(x+y), \\
\left\langle x\left|T_{0,2}\right| y\right\rangle & =i(x-y)^{10} \sum_{\ell=1}^{2} b_{02 \ell}\left(x^{\ell} y^{4-\ell}+x^{4-\ell} y^{\ell}\right) \operatorname{sign}(x-y), \\
\left\langle x\left|T_{2,0}\right| y\right\rangle & =i b_{201}(x-y)^{12} x y \operatorname{sign}(x-y),
\end{aligned}
$$

where $b_{\mu \nu \ell}$ are the real coefficients listed in Table 3. Eqs. (96) - (101) show that indeed $T_{\mu, \nu}$ and consequently $\mathcal{Q}_{3}$ are Hermitian operators. Hence in view of (22), (31) and (47), $Q_{3}$ has the following general form

$$
Q_{3}=T_{0,0}+\lambda_{1} T_{1,0}+\kappa_{1} T_{0,1}+\lambda_{1} \kappa_{1} T_{1,1}+\lambda_{1}^{2} T_{2,0}+\kappa_{1}^{2} T_{0,2}+\lambda_{3} p^{-15}-i \kappa_{3} p^{-15} \mathcal{P}
$$

where $\lambda_{1}$ and $\kappa_{1}$ are the real free parameters that fix $Q_{1}$ and $\lambda_{3}$ and $\kappa_{3}$ are a pair of arbitrary real constant coefficients.

In order to derive a manifestly Hermitian expression for $Q_{3}$, we first derive, after a lengthy calculation, the following manifestly Hermitian form of $T_{\mu, \nu}$.

$$
T_{\mu, \nu}=\left\{\begin{array}{clr}
\sum_{\ell=0}^{5} c_{\mu \nu \ell}\left\{x^{2 \ell}, \frac{1}{p^{15-2 \ell}}\right\} & \text { for } & \nu \neq 1 \\
-i \sum_{\ell=0}^{4} c_{\mu 1 \ell}\left\{x^{2 \ell}, \frac{1}{p^{15-2 \ell}}\right\} \mathcal{P} & \text { for } & \nu=1,
\end{array}\right.
$$

where $c_{\mu \nu \ell}$ are real constants given in Table 1, Substituting (103) in (102) we finally find (50). 


\section{References}

[1] A. Mostafazadeh, J. Math. Phys. 43, 2814 (2002); ibidem 43, 3944 (2002)

[2] A. Mostafazadeh, J. Math. Phys. 43, 205 (2002)

[3] M. Reed and B. Simon, Functional Analysis, vol. 1, Academic Press, San Diego, 1980

[4] A. Mostafazadeh, Preprint: quant-ph/0310164

[5] A. Mostafazadeh and A. Batal, J. Phys. A: Math. Gen. 37, 11645 (2004)

[6] A. Mostafazadeh, J. Phys. A: Math. Gen. 38, 6557 (2005)

[7] A. Mostafazadeh, J. Math. Phys. 46, 102108 (2005)

[8] A. Mostafazadeh, Nucl. Phys. B, 640, 419 (2002)

[9] A. Mostafazadeh, J. Math. Phys. 44, 974 (2003)

[10] H. B. Geyer, F. G. Scholtz, and I. Snyman, Czech J. Phys. 54, 1069 (2004)

[11] H. F. Jones, J. Phys. A: Math. Gen. 38, 1741 (2005)

[12] C. M. Bender, D. C. Brody and H. F. Jones, Phys. Rev. Lett. 89, 270401 (2002)

[13] A. Mostafazadeh, J. Phys. A: Math. Gen. 38, 3213 (2005)

[14] C. M. Bender, D. C. Brody and H. F. Jones, Phys. Rev. D 70, 025001 (2004)

[15] P. V. O'Neil, Beginning Partial Differential Equations, Wiley-Intescience, New York, 1999.

[16] C. M. Bender and S. Boettcher, Phys. Rev. Lett. 80, 5243 (1998)

[17] P. Dorey, C. Dunning, and R. Tateo, J. Phys. A: Math. Gen. 34, 5679 (2001)

[18] K. C. Shin, Commun. Math. Phys. 229, 543 (2002)

[19] A. Mostafazadeh, Czech J. Phys. 54, 1125 (2004)

[20] A. Mostafazadeh, J. Phys. A: Math. Gen. 36, 7081 (2003)

[21] M. Tegmark, Class. Quantum Grav. 14, L69 (1997)

[22] F. G. Scholtz, H. B. Geyer, and F. J. W. Hahne, Ann. Phys. (NY) 21374 (1992)

[23] A. Ashtekar, J. Lewandowski, D. Marolf, J. Mourão, and T. Thiemann, J. Math. Phys. 36, $6456(1995)$ 


\begin{tabular}{||c||c|c|c|c|c|c||}
\hline \hline$\ell$ & 0 & 1 & 2 & 3 & 4 & 5 \\
\hline \hline$c_{00 \ell}$ & $\frac{141274966833}{32}$ & $\frac{3830434839}{64}$ & $\frac{23858793}{64}$ & $\frac{43479}{32}$ & $\frac{267}{64}$ & $\frac{1}{80}$ \\
\hline$c_{01 \ell}$ & $\frac{24081603}{20}$ & $\frac{328947}{40}$ & $\frac{16327}{80}$ & $\frac{35}{48}$ & $\frac{1}{480}$ & - \\
\hline$c_{10 \ell}$ & $\frac{54563145}{16}$ & $\frac{1430535}{16}$ & $\frac{8695}{16}$ & $\frac{5}{3}$ & 0 & 0 \\
\hline$c_{11 \ell}$ & $\frac{1547}{4}$ & $\frac{61}{4}$ & $\frac{1}{12}$ & 0 & 0 & - \\
\hline$c_{02 \ell}$ & -357 & $\frac{-25}{4}$ & $\frac{1}{12}$ & 0 & 0 & 0 \\
\hline$c_{20 \ell}$ & $\frac{-2275}{4}$ & & & & 0 & 0 \\
\hline \hline
\end{tabular}

Table 1: Numerical values of the coefficients $c_{\mu \nu \ell}$ 


\begin{tabular}{||c||c|c|c|c|c|c|c|c|c|c||}
\hline \hline$\ell$ & 1 & 2 & 3 & 4 & 5 & 6 & 7 & 8 & 9 & 10 \\
\hline \hline$a_{00 \ell}$ & $\frac{2745171}{32}$ & $\frac{2745171}{32}$ & $\frac{677457}{16}$ & $\frac{439857}{32}$ & $\frac{52029}{16}$ & $\frac{9375}{16}$ & $\frac{651}{8}$ & $\frac{273}{32}$ & $\frac{5}{8}$ & $\frac{1}{40}$ \\
\hline$a_{01 \ell}$ & $\frac{70317}{5}$ & $\frac{23592}{5}$ & $\frac{20207}{20}$ & $\frac{794}{5}$ & $\frac{777}{40}$ & $\frac{217}{120}$ & $\frac{7}{60}$ & $\frac{1}{240}$ & - & - \\
\hline$a_{10 \ell}$ & $\frac{1110915}{8}$ & $\frac{363315}{8}$ & $\frac{36355}{4}$ & $\frac{9305}{8}$ & 90 & $\frac{10}{3}$ & - & - & - & - \\
\hline$a_{11 \ell}$ & $\frac{351}{2}$ & $\frac{71}{2}$ & $\frac{11}{3}$ & $\frac{1}{6}$ & - & - & - & - & - & - \\
\hline$a_{02 \ell}$ & 388 & 48 & $\frac{11}{3}$ & $\frac{1}{6}$ & - & - & - & - & - & - \\
\hline$a_{20 \ell}$ & $\frac{325}{2}$ & $\frac{25}{2}$ & - & - & - & - & - & - & - & - \\
\hline \hline
\end{tabular}

Table 2: Coefficients $a_{\mu \nu \ell}$ of the operators $T_{\mu, \nu}$ 


\begin{tabular}{||c||c|c|c|c|c||}
\hline \hline$\ell$ & 1 & 2 & 3 & 4 & 5 \\
\hline \hline$b_{00 \ell}$ & $\frac{79}{11468800}$ & $\frac{79}{2867200}$ & $\frac{533}{8601600}$ & $\frac{947}{8601600}$ & $\frac{53}{983040}$ \\
\hline$b_{01 \ell}$ & $\frac{601}{532224000}$ & $\frac{4757}{1596672000}$ & $\frac{5443}{798336000}$ & $\frac{937}{266112000}$ & - \\
\hline$b_{10 \ell}$ & $\frac{211}{18923520}$ & $-\frac{533}{63866880}$ & $\frac{9127}{510935040}$ & - & - \\
\hline$b_{11 \ell}$ & $\frac{1}{70963200}$ & $\frac{1}{383201280}$ & - & - & - \\
\hline$b_{02 \ell}$ & $-\frac{13}{479001600}$ & $\frac{15}{958003200}$ & - & - & - \\
\hline$b_{20 \ell}$ & $-\frac{1}{76640256}$ & - & - & - & - \\
\hline \hline
\end{tabular}

Table 3: Coefficients $b_{\mu \nu \ell}$ of the matrix elements $\left\langle x\left|T_{\mu, \nu}\right| y\right\rangle$ 